\title{
A Robust 3D Self-powered Photoelectrochemical type Photodetector based on MoSe2 Nanoflower
}

\author{
Kai Wang \\ Xiangtan University \\ Jie Wu \\ Xiangtan University \\ Gexiang Chen \\ Xiangtan University \\ Hui Qiao \\ Xiangtan University \\ Yang Zhou \\ Xiangtan University \\ 3176-0657 \\ Xiang Qi \\ Xiangtan University
}

Jun Li ( $\nabla$ lijun@xtu.edu.cn )

Faculty of Physics and Optoelectronic Engineering, Xiangtan University https://orcid.org/0000-0002-

\section{Research Article}

Keywords: Selenide molybdenum, Self-powered photodetector, Three-dimensional (3D) structure

Posted Date: March 24th, 2021

DOI: https://doi.org/10.21203/rs.3.rs-343198/v1

License: (1) This work is licensed under a Creative Commons Attribution 4.0 International License. Read Full License 


\section{Abstract}

Molybdenum selenide $\left(\mathrm{MoSe}_{2}\right)$ has been extensively studied in recent years due to its strong absorption for sunlight and unique band structure. Herein, a self-assembly three-dimensional (3D) $\mathrm{MoSe}_{2}$ nanoflowers were prepared by a two-step process. Significantly, the photodetection device based on $\mathrm{MoSe}_{2}$ nanoflowers exhibited a maximum responsivity about $12.39 \mathrm{~mA} / \mathrm{W}$ and a rapid photo-response time about $0.15 \mathrm{~s}$ at $0 \mathrm{~V}$ bias under simulated sunlight exposure benefiting from its large specific surface area and unique morphologic structure. Meanwhile, we demonstrated the outstanding stability after two weeks of the photodetection device. In this way, the $\mathrm{MoSe}_{2}$ nanoflower-based photodetectors enriched the basic research of molybdenum selenide and provided some reference for the following researches based on molybdenum selenide.

\section{Introduction}

The process of optical detection is a significant phenomenon of converting light into electrical signal that plays important roles in photoelectric device, chemical/biomedical sensors military and information communication[1-3]. Meanwhile, two-dimensional materials such as $\mathrm{MoS}_{2}$ [4], WSe 2 [5], $\mathrm{MoTe}_{2}$ [6], $\mathrm{MoSe}_{2}[7]$ have been proved to be promising optoelectronic materials due to their unique growth structure and excellent photoelectric properties[8]. Among $\mathrm{MeX}_{2}$ structural formula, $\mathrm{MoSe}_{2}$ has become one of the promising candidate materials for near infrared photodetectors due to few-layer $\mathrm{MoSe}_{2}$ huge advantages such as direct band gap (monolayer or less) about $1.5 \mathrm{eV}$ and high anti-photo corrosion stability, as well as the stronger absorption of sunlight [9-12]. The pretty superiority makes $\mathrm{MoSe}_{2}$ suitable for working in tough environment. Generally, most of photodetectors need to apply an external bias voltage to obtain considerable detection capability, which requires a constant power supply. Therefore, self-powered system is increasingly popular among researchers due to without external power supply, environment-friendly, low power consumption[13, 14].

Although researchers have made PDs based on nanosheets and nanofilms with wonderful performance, such as $\mathrm{GeH}$ nanosheets with excellent responsivity and rapid response time prepared by Liu et al.[15] and the ultrathin $\mathrm{MoSe}_{2}$ films with almost perfect light absorption synthetized by Du et al.[16], there are still need to search for other techniques to meliorate their performance. Recently, researchers pay special attention to the development of new semiconductor materials, new structures of compounds, providing unique solutions to enhance current transmission and improve the performance of devices[17].

Researchers designed other structures such as nanoflowers, thin of nanosheets and branched nanorods to enhance the performance of device[18-20]. The nanoflower structures have been illustrated to improve the absorption of light through multiple refraction of light and number of active regions $[21,22] . P D_{S}$ manufactured with such structure also exhibited fabulous reliability and sensitivity, low requirements and high-speed operations with quick response time[9]. Aggarwal et al. reported a self-powered photodetector based GaN flowers with increasing the active area for absorbing the incident photons due to high surface to volume ratio[7, 9]. And Song et al [23] prepared the $\mathrm{TiO}_{2}$ nanoflowers by hydrothermal method and the 
$\mathrm{TiO}_{2}$ nanoflower-based photodetectors exhibited a great self-powered performance with eximious stability and repeatability. Remarkably, the detectivity of a PD can be improved by employing a novel approach to promote light absorption [21].

In this letter,we have prepared $\mathrm{MoSe}_{2}$ nanoflowers with large specific surface area that make the absorption of incident light more adequate $[9,21]$ and large absorption area of incident photons and strong detection ability[23, 24], which was a kind of three-dimensional (3D) nanostructure [25, 26]. In addition, Under the condition of global energy crisis, the independent and sustainable self-powered supply system is a necessary issue that arouses attention of researchers. The self-powered photodetectors presented the advantages of low power consumption and energy saving, which are very suitable for extreme conditions[27-30]. The PDs based on $\mathrm{MoSe}_{2}$ nanoflowers showed an obvious performance such as the impressive responsivity and rapid response time at zero bias with long-term stability and repeatability under simulated sunlight exposure in $\mathrm{KOH}$ electrolyte solution. Accordingly, it also provides some references for the exploration of $\mathrm{MoSe}_{2}$ in the future.

\section{Experimental Section}

\subsection{Synthesis of $\mathrm{MoSe}_{2}$ Nanoflower}

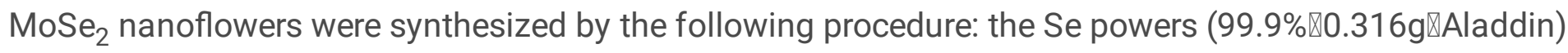
with $10 \mathrm{ml}$ Hydrazine hydrate (80wt. \%区Hunan Hui Hong Reagent Co., Ltd.) and Sodium molybdate dihydrate (99.8\% $\$ 0.484 \mathrm{~g} \otimes$ Shanghai Macklin Biochemical Co., Ltd.) dissolved in a $100 \mathrm{ml}$ beaker and then add a mixture of ethanol and water mixture of deionized water $(10 \mathrm{~mL})$ and alcohol $(15 \mathrm{~mL})$ under continuous agitation. The mixed solution was then moved to a $50 \mathrm{ml}$ polytetrafluoroethylene lined stainless steel autoclave. The autoclave was sealed and kept at $200^{\circ} \mathrm{C}$ for 24 hours. The prepared black precipitates were collected with a $50 \mathrm{ml}$ centrifuge tube and then centrifuged with ethanol for at least three times followed by drying at $60{ }^{\circ} \mathrm{C}$ for $10 \mathrm{~h}$ in vacuum. Finally, the centrifuged products were annealed in a Chemical Vapor Deposition (CVD) tubular furnace at $600{ }^{\circ} \mathrm{C}$ for 2 hours. The preparation process of MoSe2 nanoflower was presented in Fig. 1.

\subsection{Characterizations of $\mathrm{MoSe}_{2}$ nanoflower}

The X-ray diffraction (XRD) shows the characteristic peaks of $\mathrm{MoSe}_{2}$ nanoflowers with $\mathrm{Cu}$ Ka radiation at a scanning rate of $1.5^{\circ} \mathrm{min}-1$, which is compatible with the standard peaks. Raman microscope (Renishaw, In Via) was employed further observed the crystal structure at room temperature. Moreover, the microstructures of $\mathrm{MoSe}_{2}$ nanoflowers was viewed by the Scanning Electron Microscope (SEM, JEOL, JSM-6360).

\subsection{Photo-response performance measurement}


The prepared materials were weighed $1 \mathrm{mg}$ and put into a $5 \mathrm{ml}$ small centrifuge tube. Then, $1 \mathrm{ml} \mathrm{N}$-methyl-2pyrrolidone was added to ultrasonic until the mixture was uniform. Coating the dispersed mixed solution onto indium-tin oxide (ITO) conductive glass as working electrode. The opposite electrode and reference electrode respectively chose Pt electrode and saturated calomel electrode. The three electrodes were immersed in $\mathrm{KOH}$ electrolyte solution. A 150W xenon lamp (280 980nm) was used as the light source. In addition, the photocurrent tested was recorded the electrochemistry workstation CHI660D (Chen Hua, China). Finally, we promise that all measurements were conducted under the same environmental conditions.

\section{Results And Discussion}

In Fig. 2(a), the XRD patterns of the prepared $\mathrm{MoSe}_{2}$ samples were shown. It is evident that the $\mathrm{MoSe}_{2}$ nanoflowers prepared mainly grew along the [002], [004], [100], [103], [105], [008], [200], [203], [118] direction. These peaks are consistent with the standard $\mathrm{MoSe}_{2}$ diffraction peaks. To further ensure the

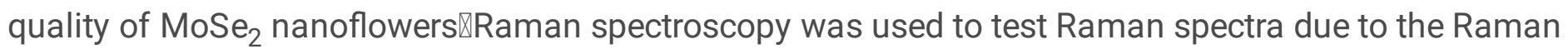
measurements is considered to be an effective method to analyze the microstructure of nanomaterials all the time [31]. As clearly seen in Fig. 2(b), the prepared $\mathrm{MoSe}_{2}$ nanoflower exhibits three characteristic peaks in the spectral range of $200-400 \mathrm{~cm}^{-1}$, which are respectively assigned to out-of-plane $\mathrm{A}_{1 \mathrm{~g}}$, in-plane and Raman active modes. The three characteristic peaks located at 241.2, 288.1 and $355.6 \mathrm{~cm}^{-1}$ that are consistent with previous reports[32, 33]. The Fig. 2(c) illustrates the $\mathrm{MoSe}_{2}$ nanoflower with the crystal structure of the top view. The SEM images of Fig. 2(d) presents shows its structural characteristics more intuitively.

Considering the light response characteristics is an essential factor for detector. To gain the photoresponse properties of $\mathrm{MoSe}_{2}$ nanoflowers, the responsivity switching behavior of the electrode coated ITO materials under simulated sunlight illumination was studied by using a photoelectrochemical (PEC) test system. The schematic diagram of optical response test was shown in Fig. 3(a). The further mechanism diagram was confirmed in Fig. 3(b). As can be seen, with $\mathrm{MoSe}_{2}$ dripping onto ITO contact with the $\mathrm{KOH}$ electrolyte, electrons will immediately flow from the photoanode to the electrolyte, leaving a hole where the electrons will accumulate on the side near the electrolyte and form a space charge layer. As electrons move to dynamic equilibrium, a built-in electric field is created. When incident light hits the photoanode, corresponding electron hole pairs are generated. The internal electric field drives the electrons through the external circuit to the opposite electrode, and then through the platinum electrode to the electrolyte.

Generally, while low mobility of carriers in electrolyte results in the low responsivity of PEC-type photodetectors, its unique three electrode structure design is incomparable to other types of detectors. Especially, they can provide power with themselves without external power supply. During the photoelectrochemical test, we evaluated the photo-response performance of the $\mathrm{MoSe}_{2}$ nanoflower-based photodetection device with simulated sunlight illumination. The Fig. 4 illustrated the photodetector gained a great photo-response performance at different power intensity especially in the case of $0 \mathrm{~V}$. Clearly, the 
photocurrent density showed a trend of near linear growth with different power intensities, which reached $0.47 \mu \mathrm{A} \mathrm{cm}^{-2}$ at $60 \mathrm{~mW} \mathrm{~cm}^{-2}$ and photocurrent intensity was up to a maximum of $1.74 \mu \mathrm{A} \mathrm{cm}^{-2}$ for incident light power $140 \mathrm{~mW} / \mathrm{cm}^{2}$ at $0 \mathrm{~V}$ bias. This can be attributed to the accelerated separation of photogenerated carriers at higher power densities. Besides that, the flower-like structure increased active regions resulting more enough absorption of light and accelerating the efficiency of current transmission, which may be beneficial for the large photocurrent density. Notably, the photo-response at $\mathrm{OV}$ certified the $\mathrm{MoSe}_{2}$ nanoflower-based photodetector working normally without external bias. In addition, $\mathrm{MoSe}_{2}$ nanoflowerbased photodetector exhibited a competitive performance such as the eximious responsivity and the rapid response time, compared with other PEC-type photodetectors (See Table 1 for details) [34-37].

\section{Table 1}

The performance comparison with other photodetection device.

\begin{tabular}{|c|c|c|c|c|c|c|}
\hline Material & $\begin{array}{l}\text { Measurement } \\
\text { type and } \\
\text { condition }\end{array}$ & Wavelength & Responsivity & $\begin{array}{l}\text { photocurrent } \\
\text { density at } \\
\text { OV }\end{array}$ & $\begin{array}{l}\text { Response } \\
\text { time }\end{array}$ & Reference \\
\hline $\begin{array}{l}\text { GaN } \\
\text { nanowires }\end{array}$ & $P E C, V=0 V$ & $365 \mathrm{~nm}$ & $322.5 \mu \mathrm{A} / \mathrm{W}$ & $12.9 \mu \mathrm{Acm}^{-2}$ & $0.28 \mathrm{~s}$ & [38] \\
\hline $\begin{array}{l}\text { Te@Se } \\
\text { nanotubes }\end{array}$ & $\begin{array}{l}P E C, V=0.6 V \\
0.5 M ~ K O H\end{array}$ & $365-700 \mathrm{~nm}$ & $98.8 \mu \mathrm{A} / \mathrm{W}$ & $7.79 \mu \mathrm{Acm}^{-2}$ & $0.52 \mathrm{~s}$ & [34] \\
\hline $\begin{array}{l}\text { BP } \\
\text { nanosheets }\end{array}$ & $\begin{array}{l}P E C, V=0 V \\
0.1 M ~ K O H\end{array}$ & $365-546 \mathrm{~nm}$ & $1.9-2.2 \mu \mathrm{A} / \mathrm{W}$ & $265 \mathrm{nA} \mathrm{cm}^{-2}$ & $0.5 \mathrm{~s}$ & [35] \\
\hline $\begin{array}{l}\mathrm{NiPS}_{3} \\
\text { flakes }\end{array}$ & $\begin{array}{l}\mathrm{PEC}, \mathrm{V}=0 \mathrm{~V}, \\
0.1 \mathrm{M} \mathrm{KOH}\end{array}$ & white & $3.79 \mu \mathrm{A} / \mathrm{W}$ & $724 \mathrm{nAcm}^{-2}$ & $5.8 \mathrm{~s}$ & [37] \\
\hline $\begin{array}{l}\text { InSe } \\
\text { nanosheets }\end{array}$ & $\begin{array}{l}\mathrm{PEC}, \mathrm{V}=0 \mathrm{~V}, \\
0.2 \mathrm{M} \mathrm{KOH}\end{array}$ & white & $4.9 \mu \mathrm{A} / \mathrm{W}$ & 15.9 nAcm $^{-2}$ & $5 s$ & [39] \\
\hline $\begin{array}{l}\mathrm{MoSe}_{2} \\
\text { nanoflower }\end{array}$ & $\begin{array}{l}P E C, V=0 V, \\
0.2 \mathrm{M} \mathrm{KOH}\end{array}$ & white & $12.39 \mu \mathrm{A} / \mathrm{W}$ & $1.75 \mu \mathrm{Acm}^{-2}$ & $0.15 \mathrm{~s}$ & This work \\
\hline
\end{tabular}

Importantly, the responsivity $\left(R_{h}\right)$ and the response time are the essential parameters for the photodetection device, which introduced to evaluate the functional relationship between photocurrent density and light power intensity. The $R_{h}$ obtained used the formula: $R_{h}=1 / J_{\text {light }}$, where $J_{\text {light }}$ presented the power density and I was the photocurrent density. Introducing rise time $\left(t_{r}\right)$ and decay time $\left(t_{d}\right)$ were used to assessment the photo-response behaviors of the device. The $t_{r}$ and $t_{d}$ were defined to delegate the time interval of the rising (falling) time from $10 \%(90 \%)$ to $90 \%$ (10\%), which were 0.15 s and 0.1 s, respectively. The response and recovery time were much more competitive compared with other photodetectors (Table 1 showed the detailed data). As shown in Fig. 4(a), the photocurrent density at OV bias was incisively illustrated. And the illustration in Fig. 4(a) showed a schematic diagram of the response time and the relaxation time, selected one cycle from $100 \mathrm{~mW} / \mathrm{cm}^{2}$ light response at $0 \mathrm{~V}$. The electrolyte concentration as 
an indispensable factor for the change of the photocurrent which must be consideration. The variation of photocurrent density at different concentrations can be intuitively seen from Fig. 4(b). Moreover, for the light response at OV obtained in Fig. 4(a), we have run further tests, and the results were shown in Fig. 4(c). The photocurrent density slightly increased with the increase of the optical power. The near linear growth result which was in line with expectations due to the separation of electron hole pairs and the current transmission are closely related to the power separation of electron-hole pairs and the current transmission are closely related to the power. Then, the relationship between the photocurrent density and the light response was exhibited in Fig. 4(d). In Fig. 4(d), the responsivity increased from 7.5 $\mu \mathrm{A} / \mathrm{W}$ to $12.39 \mu \mathrm{A} / \mathrm{W}$ at $0 \mathrm{~V}$ when the incident light power intensity increased from $60 \mathrm{~mW} / \mathrm{cm}^{2}$ to $140 \mathrm{~mW} / \mathrm{cm}^{2}$. Meanwhile, the increase of electrolyte concentration will lead to the increase of photocurrent density from $0.1 \mathrm{M}$ to $0.5 \mathrm{M}$ because a high concentration of electrolyte can provide a relatively large number of conducting ions, in accordance with the EIS map of the Fig. 4(e). However, the less conductive ions in the lower electrolyte concentration have little influence on the carrier flow[40]. On top of that, in order to better understand the optical response of the device at different bias and more intuitively understand the performance of the detector. We showed the LSV curves under light and dark conditions in Fig. 5(a). And then, the photocurrent density at different bias was shown in Fig. 5(b). Clearly, the photocurrent density reached $1.088 \mu \mathrm{A} / \mathrm{cm}^{2}$ at $0 \mathrm{~V}$ and attained $19.23 \mu \mathrm{A} / \mathrm{cm}^{2}$ at $1 \mathrm{~V}$ when the light power was $100 \mathrm{~mW} / \mathrm{cm}^{2}$. The change of photocurrent density from $0 \mathrm{~V}$ to $1 \mathrm{~V}$ may be due to the large specific surface area of $\mathrm{MoSe}_{2}$ nanoflower reduces the scattering of light and increases the refraction of light, which leads to the full absorption of light, which is also good for the increase of photocurrent. In addition, the large specific surface area and high light absorption are sufficient to achieve charge exchange between the holes of $\mathrm{MoSe}_{2}$ surface with the $\mathrm{OH}^{-}\left(\mathrm{h}^{+}\right.$ $\left.+\mathrm{OH}^{-}=\mathrm{OH} \cdot\right)$ in the electrolyte[41].

More importantly, stability as a basic parameter to measure the performance of photodetectors must be involved, which is a more comprehensive assessment for the performance of the device. Here, the cycle stability and time stability were examined in $0.5 \mathrm{M} \mathrm{KOH}$ electrolyte. In Fig. 6 (a), the LSV curve after 100 cycles did not decrease significantly compared with the original one, which proves the great cycle stability of the detector. In addition, to measure the time stability of $\mathrm{MoSe}_{2}$ nanoflower based photodetectors at $\mathrm{OV}$ bias, the long-time stability of the test up to 1000s is explained in Fig. 6(b) and the obvious NO/OFF switching signals was exhibited in Fig. 6(c) after the test. After 1000s of continuous operation, the photodetector demonstrated greater potential in long-term measurement as the photocurrent continues to increase. Finally, the Fig. 6(d) exhibited the stability in two weeks. Obviously, although the photocurrent was slightly reduced, it still maintained the pretty performance. Generally, the excellent stability provides a solid foundation for the research of $\mathrm{MoSe}_{2}$ nanoflower-based photodetector.

\section{Conclusion}

In summary, we have successfully prepared the $\mathrm{MoSe}_{2}$ nanoflower by a two-step method and fabricated a photodetector based on $\mathrm{MoSe}_{2}$ nanoflowers with decent performances. The Raman and XRD measurements illustrated the great crystallinity of the $\mathrm{MoSe}_{2}$ nanoflower. It was applied PEC-type 
photodetector and showed a self-powered performance with $\mathrm{KOH}$, such as the short photo-response time and the high photocurrent density and outstanding responsivity at $\mathrm{OV}$ bias. In addition, the $\mathrm{MoSe}_{2}$ nanoflower-based photodetectors gained excellent cycle stability and time stability in $0.5 \mathrm{M} \mathrm{KOH}$ electrolyte solution, respectively. This work provides an effective way to study $\mathrm{MoSe}_{2}$ nanoflower-based PEC-type selfpowered detectors.

\section{Declarations}

\section{Acknowledgements}

This work was financially supported by the Open Fund based on innovation platform of Hunan colleges and universities (No. 19K095), the Grants from National Natural Science Foundation of China (No. 11874316), the Program for Changjiang Scholars and Innovation Research Team in University (IRT 17R91).

\section{Data Availability}

The data that support the findings of this study are available from the corresponding author upon reasonable request.

\section{Conflict of Interest}

The manuscript has no conflicts of interest and all authors agree to the publication of the paper.

\section{References}

1. Li, Z., et al., High-Performance Photo-Electrochemical Photodetector Based on Liquid-Exfoliated FewLayered InSe Nanosheets with Enhanced Stability. Advanced Functional Materials, 2018. 28(16): p. 1705237.

2. Liu, C.-H., et al., Graphene photodetectors with ultra-broadband and high responsivity at room temperature. Nature Nanotechnology, 2014. 9(4): p. 273-278.

3. Yin, Z., et al., Single-layer MoS2 phototransistors. 2012. 6(1): p. 74-80.

4. Cai, Z., et al., Dual-Additive Assisted Chemical Vapor Deposition for the Growth of Mn-Doped 2D MoS2 with Tunable Electronic Properties. 2020. 16(15): p. 1903181.

5. Nguyen, D.A., et al., Highly Enhanced Photoresponsivity of a Monolayer WSe2 Photodetector with Nitrogen-Doped Graphene Quantum Dots. ACS Applied Materials \& Interfaces, 2018. 10(12): p. 1032210329.

6. Wang, F., et al., 2D Metal Chalcogenides for IR Photodetection. 2019. 15(30): p. 1901347. 
7. Jung, C., et al., Highly Crystalline CVD-grown Multilayer MoSe2 Thin Film Transistor for Fast Photodetector. Scientific Reports, 2015. 5(1): p. 15313-15313.

8. Ronchi, R.M., J.T. Arantes, and S.F. Santos, Synthesis, structure, properties and applications of MXenes: Current status and perspectives. Ceramics International, 2019. 45(15): p. 18167-18188.

9. Aggarwal, N., et al., A Highly Responsive Self-Driven UV Photodetector Using GaN Nanoflowers. Advanced electronic materials, 2017. 3(5): p. 1700036.

10. Xia, Y., et al., Tuning Electrical and Optical Properties of MoSe2 Transistors via Elemental Doping. Advanced Materials Technologies, 2020. 5(7): p. 2000307.

11. Wang, L., et al., Laser Annealing Improves the Photoelectrochemical Activity of Ultrathin MoSe2 Photoelectrodes. ACS Applied Materials \& Interfaces, 2019. 11(21): p. 19207-19217.

12. Chen, X., et al., Hollow Spherical Nanoshell Arrays of 2D Layered Semiconductor for High-Performance Photodetector Device. Advanced Functional Materials, 2018. 28(8): p. 1705153.

13. Wang, X., et al., Light-Triggered Pyroelectric Nanogenerator Based on a pn-Junction for Self-Powered Near-Infrared Photosensing. ACS Nano, 2017. 11(8): p. 8339-8345.

14. Shen, L., et al., A Self-Powered, Sub-nanosecond-Response Solution-Processed Hybrid Perovskite Photodetector for Time-Resolved Photoluminescence-Lifetime Detection. Advanced Materials, 2016. 28(48): p. 10794-10800.

15. Liu, N., et al., Hydrogen Terminated Germanene for a Robust Self-Powered Flexible Photoelectrochemical Photodetector. Small, 2020. 16(23): p. 2000283.

16. Du, W., et al., An ultrathin MoSe2 photodetector with near-perfect absorption. Nanotechnology, 2020. 31(22): p. 225201.

17. Al-Gaashani, R., et al., XPS and structural studies of high quality graphene oxide and reduced graphene oxide prepared by different chemical oxidation methods. Ceramics International, 2019. 45(11): p. 14439-14448.

18. Zhang, J., et al., $1 T @ 2 H-M o S e 2$ nanosheets directly arrayed on Ti plate: An efficient electrocatalytic electrode for hydrogen evolution reaction. Nano Research, 2018. 11(9): p. 4587-4598.

19. Najafi, L., et al., Doped-MoSe2 Nanoflakes/3d Metal Oxide-Hydr(Oxy)Oxides Hybrid Catalysts for pHUniversal Electrochemical Hydrogen Evolution Reaction. Advanced Energy Materials, 2018. 8(27): p. 1801764.

20. Deng, S., et al., Phase Modulation of (1T-2H)-MoSe2/TiC-C Shell/Core Arrays via Nitrogen Doping for Highly Efficient Hydrogen Evolution Reaction. Advanced Materials, 2018. 30(34): p. 180222.

21. Xiao, D., et al., Atomic-Scale Intercalation of Graphene Layers into MoSe2 Nanoflower Sheets as a Highly Efficient Catalyst for Hydrogen Evolution Reaction. ACS Applied Materials \& Interfaces, 2020. 12(2): p. 2460-2468.

22. Huang, Y., et al., LiCl-CN nanotubes ceramic films with highly efficient visible light - Driven photocatalytic active for bisphenol A degradation and efficient regeneration process. Ceramics International, 2020. 46(17): p. 26492-26501. 
23. Song, Z., et al., The synthesis of TiO2 nanoflowers and their application in electron field emission and self-powered ultraviolet photodetector. Materials Letters, 2016. 180: p. 179-183.

24. Gil-González, E., et al., Control of experimental conditions in reaction flash-sintering of complex stoichiometry ceramics. Ceramics International, 2020. 46(18, Part B): p. 29413-29420.

25. Xing, X., et al., Room temperature ferromagnetism and its origin for amorphous MoSe2 nanoflowers. Applied Physics Letters, 2018. 112(12): p. 122407.

26. Zhao, Y., et al., Enhanced optical reflectivity and electrical properties in perovskite functional ceramics by inhibiting oxygen vacancy formation. Ceramics International, 2020.

27. Han, W., et al., Atomically Thin Oxyhalide Solar-Blind Photodetectors. Small, 2020. 16(23): p. 2000228.

28. Li, J., et al., High-Performance, Self-Powered Photodetectors Based on Perovskite and Graphene. ACS Applied Materials \& Interfaces, 2017. 9(49): p. 42779-42787.

29. Chowdhury, A.M., et al., Self-Powered, Broad Band, and Ultrafast InGaN-Based Photodetector. ACS Applied Materials \& Interfaces, 2019. 11(10): p. 10418-10425.

30. Liu, H., et al., Self-Powered Broad-band Photodetectors Based on Vertically Stacked WSe2/Bi2Te3 $p-n$ Heterojunctions. ACS Nano, 2019. 13(11): p. 13573-13580.

31. Wang, B., et al., One-pot synthesized Bi2Te3/graphene for a self-powered photoelectrochemical-type photodetector. Nanotechnology, 2019. 31(11): p. 115201.

32. Abderrahmane, A., et al., High photosensitivity few-layered MoSe2 back-gated field-effect phototransistors. Nanotechnology, 2014. 25(36): p. 365202.

33. Dai, T., et al., Layer-controlled synthesis of wafer-scale MoSe2 nanosheets for photodetector arrays. Journal of Materials Science, 2018. 53(11): p. 8436-8444.

34. Huang, W., et al., Enhanced Photodetection Properties of Tellurium@Selenium Roll-to-Roll Nanotube Heterojunctions. Small, 2019. 15(23): p. 1900902.

35. Ren, X., et al., Environmentally Robust Black Phosphorus Nanosheets in Solution: Application for SelfPowered Photodetector. Advanced Functional Materials, 2017. 27(18): p. 1606834.

36. Ng, S., et al., Photoelectrochemical ultraviolet photodetector by anodic titanium dioxide nanotube layers. Sensors and Actuators A: Physical, 2018. 279: p. 263-271.

37. Liu, J., et al., A Robust 2D Photo-Electrochemical Detector Based on NiPS3 Flakes. Advanced Electronic Materials, 2019. 5(12): p. 1900726.

38. Zhang, M., et al., High performance self-powered ultraviolet photodetectors based on electrospun gallium nitride nanowires. Applied Surface Science, 2018. 452: p. 43-48.

39. Li, Z., et al., High-Performance Photo-Electrochemical Photodetector Based on Liquid-Exfoliated FewLayered InSe Nanosheets with Enhanced Stability. Advanced Functional Materials, 2018. 28(16): p. 1705237.

40. Ren, X., et al., Photodetectors: Environmentally Robust Black Phosphorus Nanosheets in Solution: Application for Self-Powered Photodetector (Adv. Funct. Mater. 18/2017). 2017. 27(18). 
41. Chen, K., et al., Photoelectrochemical Self-Powered Solar-Blind Photodetectors Based on Ga2O3 Nanorod Array/Electrolyte Solid/Liquid Heterojunctions with a Large Separation Interface of Photogenerated Carriers. ACS Applied Nano Materials, 2019. 2(10): p. 6169-6177.

\section{Figures}

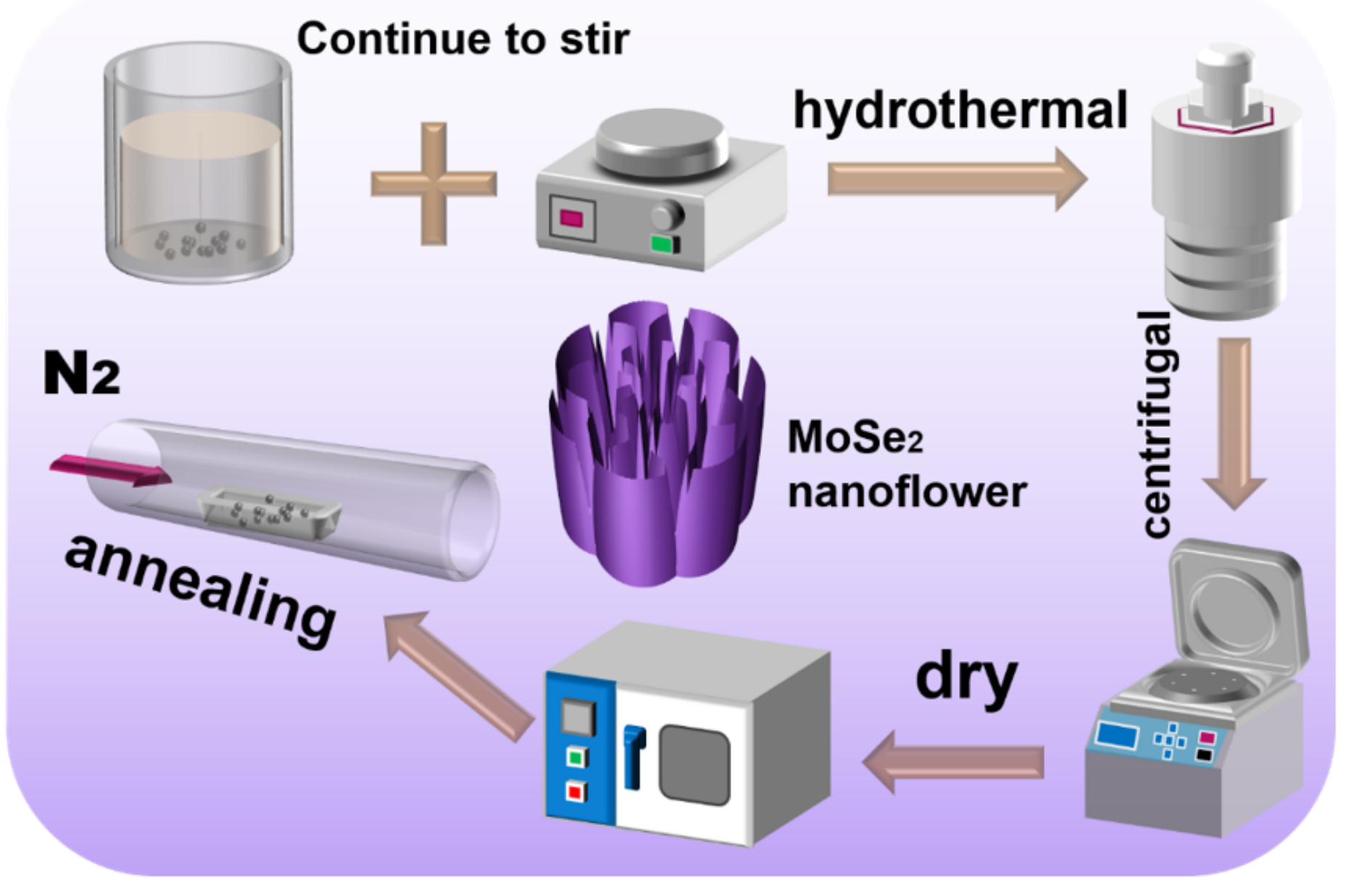

Figure 1

Schematic diagram of preparation process of Molybdenum selenide (MoSe2) nanoflower. 

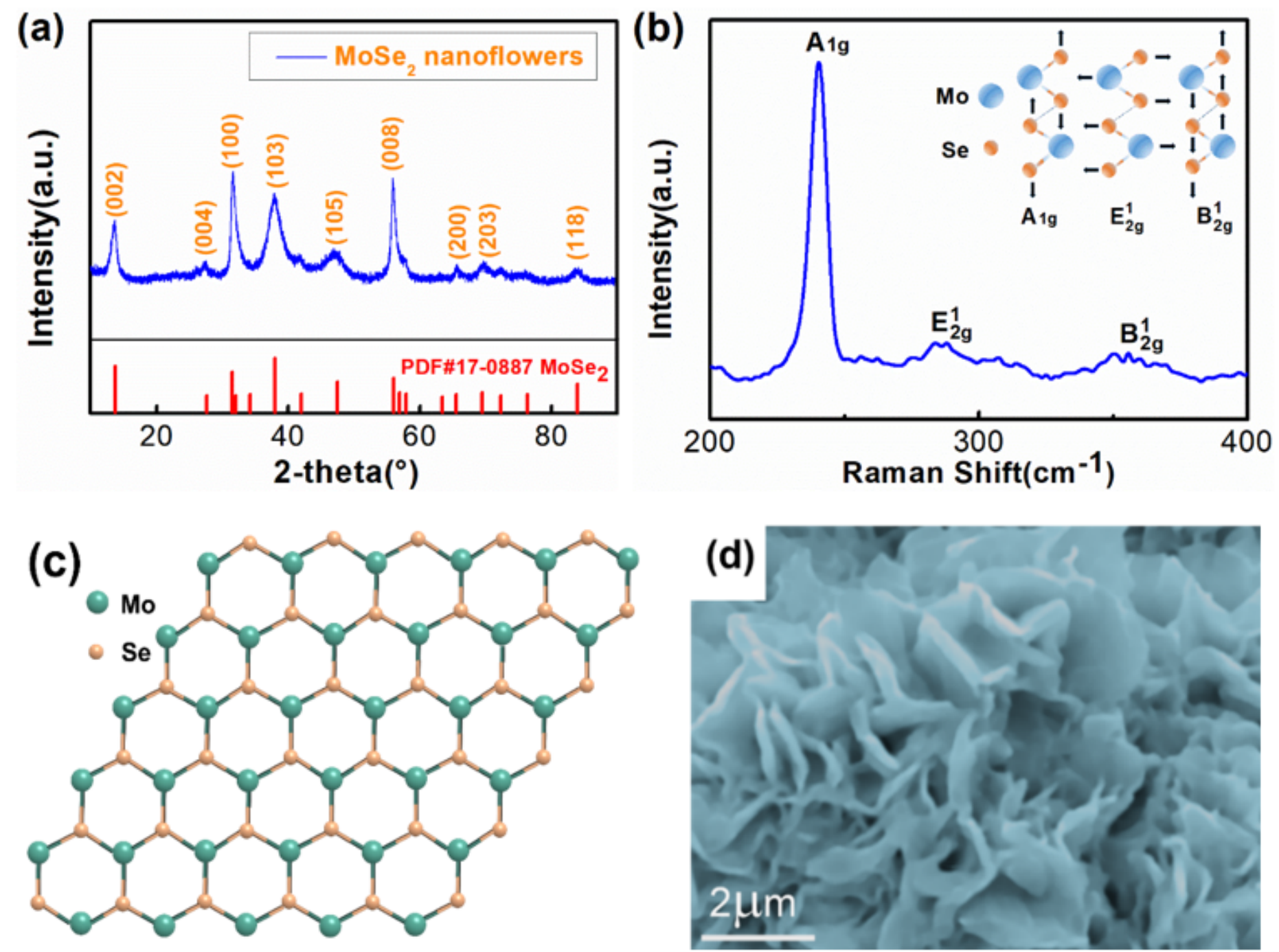

Figure 2

(a) The XRD patterns of MoSe2 nanoflower (b) The Raman spectra of MoSe2 nanoflower and the illustration shows the Raman vibration pattern. Blue and orange balls represent Mo and Se atoms $\llbracket$ respectively. (c) Top view of MoSe2 nanoflower atomic structure model. The cyan and yellow spheres represent molybdenum and selenium atoms, respectively. (d) SEM image of MoSe2 nanoflower. 
(a)

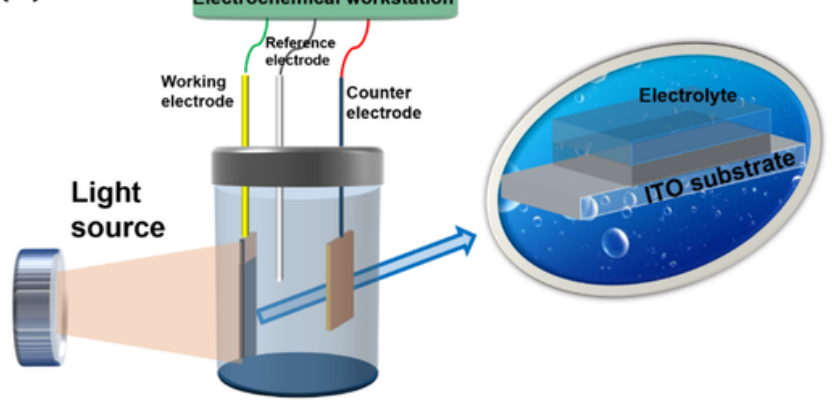

(b)

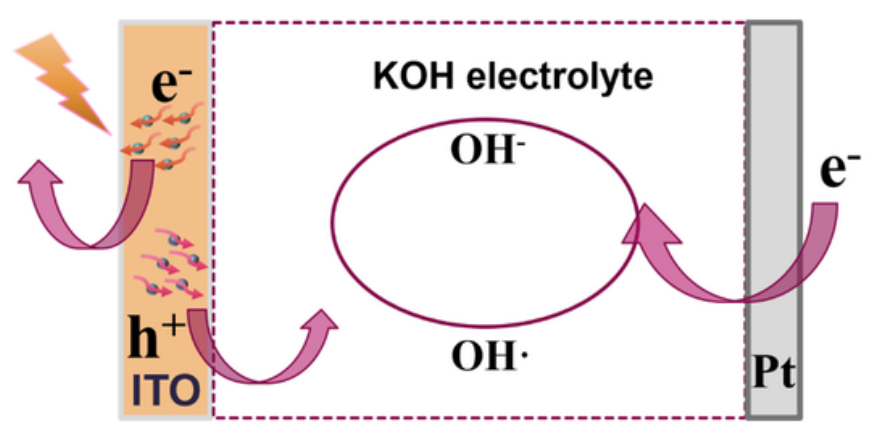

\section{Figure 3}

(a)The schematic diagram of the optical response performance test with the conventional three-electrodes photoelectrochemical test system and (b) the further mechanism reaction diagram. 
(a)

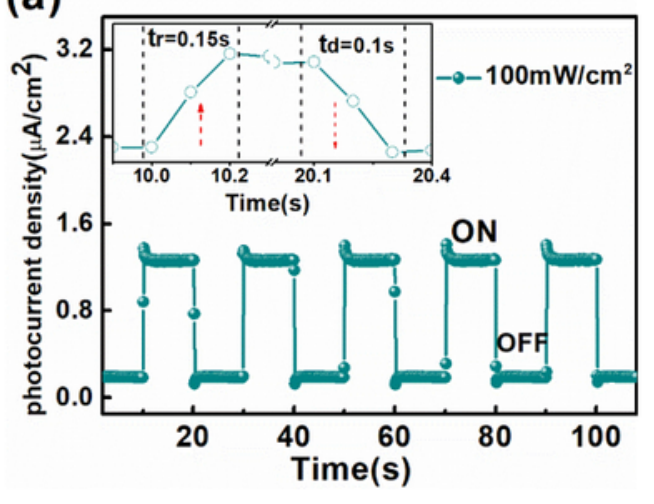

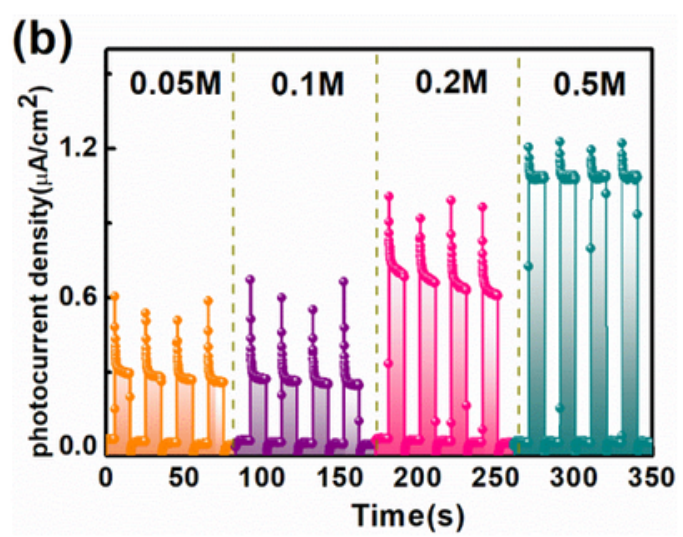

(c)
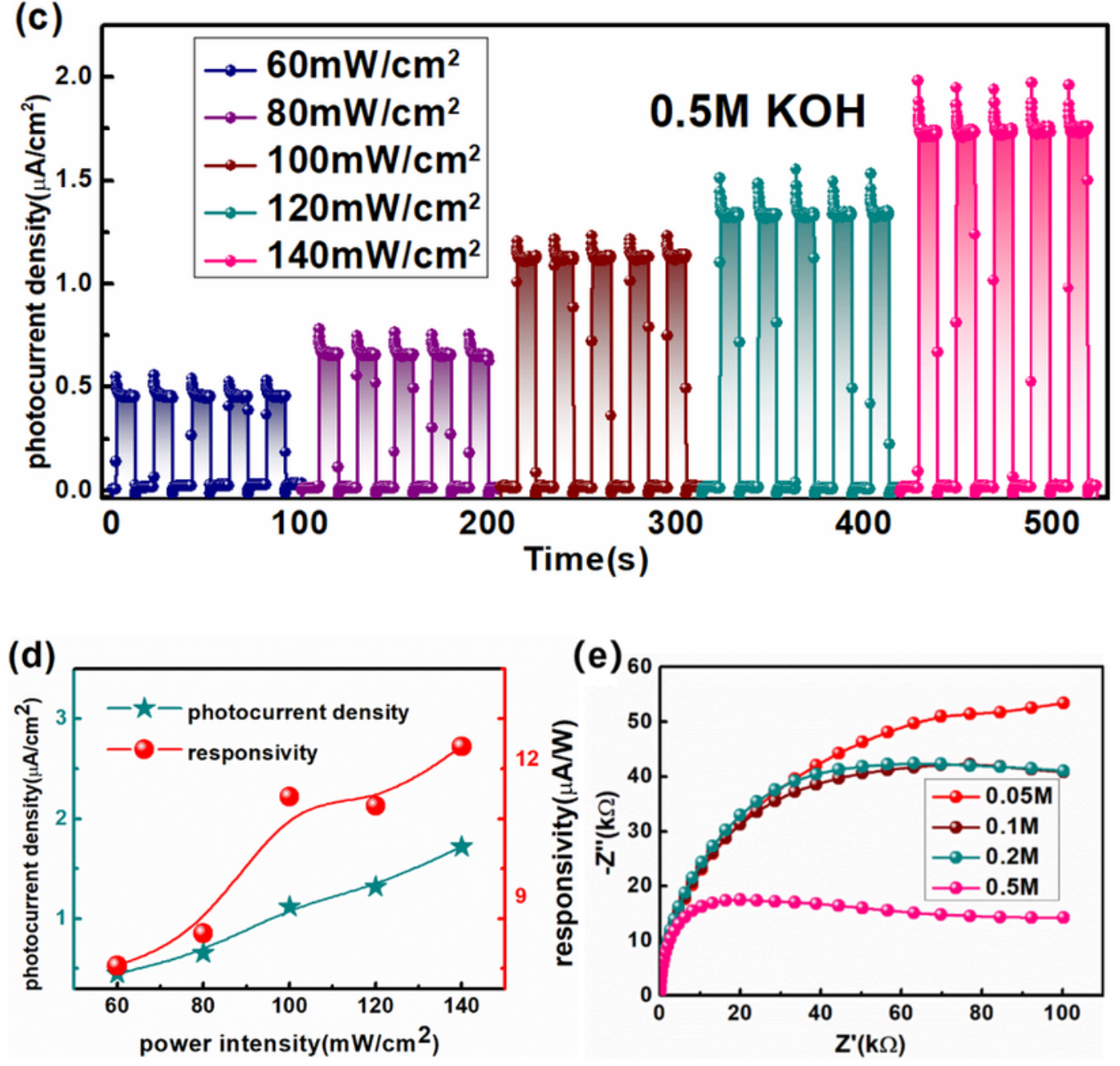

Figure 4

(a)The photocurrent density within five cycles at zero potential under simulated sunlight and the illustration shows the response time. (b) The photocurrent density curves at different concentrations. (c)The photocurrent density curve at different power with $0.5 \mathrm{M} \mathrm{KOH}$. (d) The photocurrent density and optical responsivity at different power. (e) The EIS map of different $\mathrm{KOH}$ electrolyte concentration $(0.05 \mathrm{M}$, $0.1 \mathrm{M}, 0.2 \mathrm{M}, 0.5 \mathrm{M})$. 
(a)

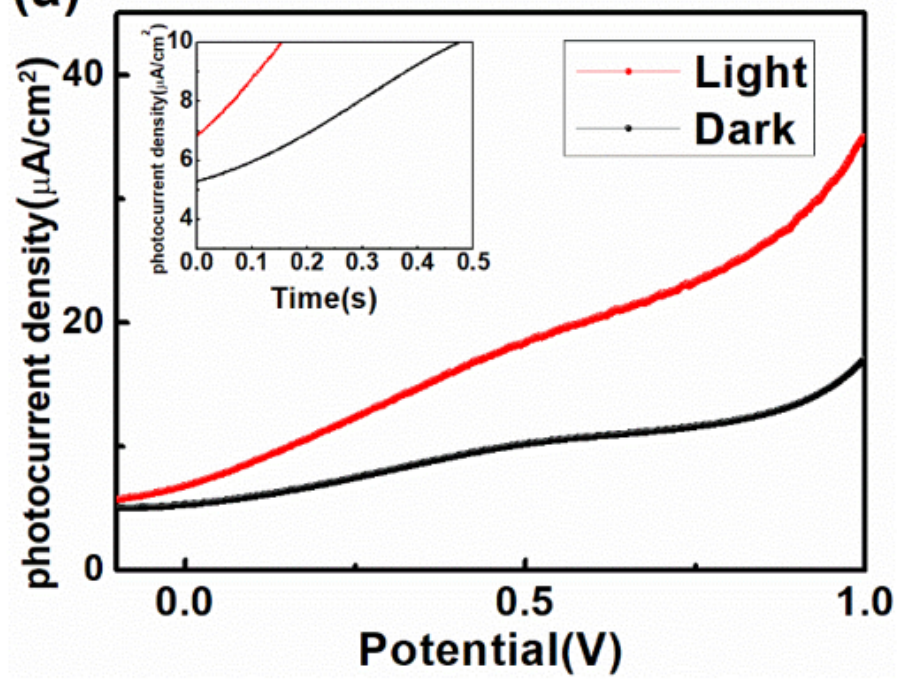

(b)

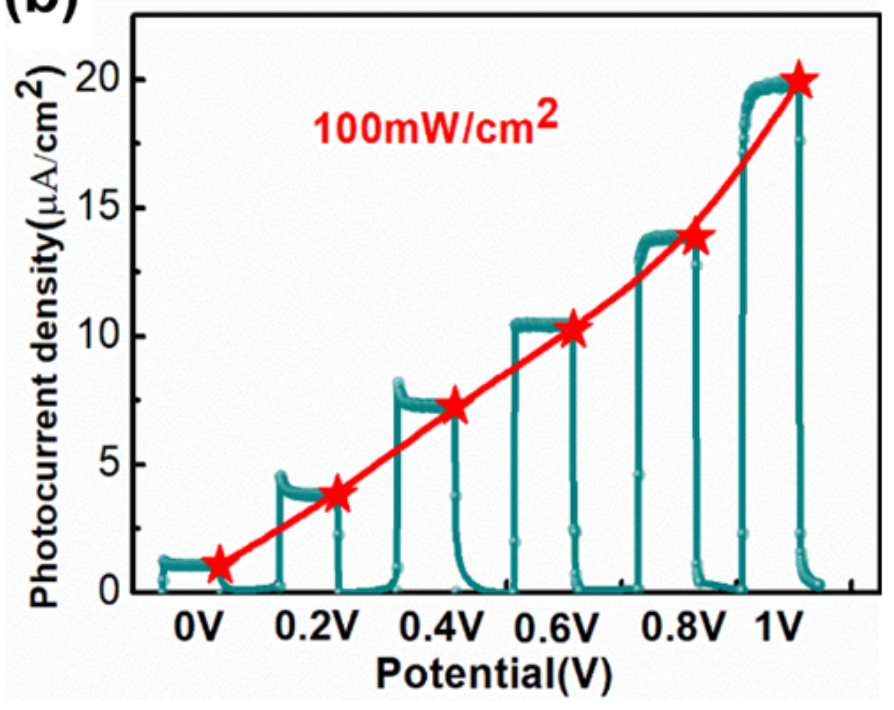

Figure 5

(a) The LSV curves of light and dark. (b) the photocurrent density at different bias from OV to 1V. 

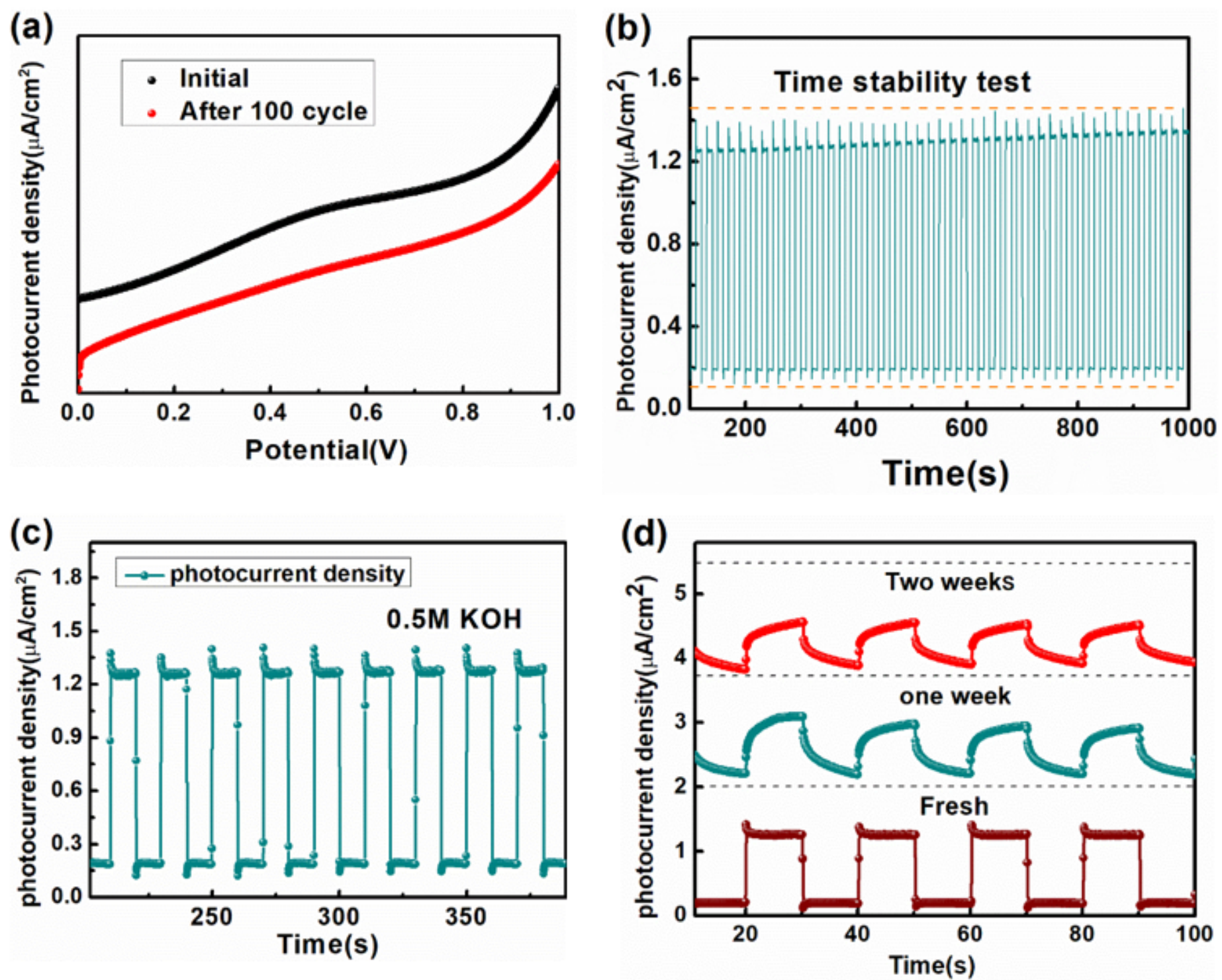

Figure 6

(a) Stability within 100 cycles. (b) Testing stability within 1000s. (c) The obvious switching signal from the (b). (d) Time stability in two weeks. 